\title{
The magnetic system SMSS J1606-1000 as a period bouncer
}

\author{
Adela Kawka, ${ }^{1 \star}$ Stéphane Vennes, ${ }^{2}$ Lilia Ferrario, ${ }^{2}$ M.S. Bessell, ${ }^{3}$ S.C. Keller, ${ }^{3}$ E. Paunzen, ${ }^{4}$ \\ D.A.H. Buckley, ${ }^{5,6}$ D. Groenewald, ${ }^{5,7}$ J. Janík ${ }^{4}$ and M. Zejda ${ }^{4}$ \\ ${ }^{1}$ International Centre for Radio Astronomy Research - Curtin University, GPO Box U1987, Perth, WA 6845, Australia \\ ${ }^{2}$ Mathematical Sciences Institute, The Australian National University, ACT 0200, Australia \\ ${ }^{3}$ Research School of Astronomy and Astrophysics, The Australian National University, Canberra, ACT 2611, Australia \\ ${ }^{4}$ Department of Theoretical Physics and Astrophysics, Masaryk University, Kotlářská 2, CZ-611 37, Czech Republic \\ ${ }^{5}$ South African Astronomical Observatory, Observatory Road, Observatory 7935, South Africa \\ ${ }^{6}$ Department of Astronomy, University of Cape Town, Rondebosch 7770, Cape Town, South Africa \\ ${ }^{7}$ South African Large Telescope, PO Box 9, Observatory 7935, South Africa
}

Accepted XXX. Received YYY; in original form ZZZ

\begin{abstract}
We report the discovery of a rare close binary system, SMSS J160639.78-100010.7, comprised of a magnetic white dwarf with a field of about $30 \mathrm{MG}$ and a brown dwarf. We measured an orbital period of $92 \mathrm{~min}$ which is consistent with the photometric period. Minimum and maximum light occur at the orbital quadratures $\Phi=0.25$ and 0.75 , respectively, and cannot be caused by reflection on the brown dwarf, but, instead, by a spot on the synchronously rotating magnetic white dwarf. The brown dwarf does not fill its Roche lobe and the system may be in a low-accretion state or, more likely, in a detached state following episodes of mass transfer. SMSS J160639.78-100010.7 is the nearest known magnetic white dwarf plus brown dwarf system.
\end{abstract}

Key words: stars: individual: SMSS J1606-1000 - stars: magnetic field - binaries: spectroscopic - white dwarfs

\section{INTRODUCTION}

White dwarfs are often found in close binaries with low mass mainsequence companions (usually M dwarf) although their number drops sharply for companions later than an M5 type (Ferrario 2012) and only a handful of white dwarfs has been found in either interacting or detached binaries containing a brown dwarf. Such systems can be detected through infrared excess and recent surveys have identified several candidates (e.g., Steele et al. 2011; Girven et al. 2011; Hogg et al. 2020). Steele et al. (2011) estimated that only 0.5 per cent of white dwarfs have a brown dwarf companion.

Only a few binary systems comprising a magnetic white dwarf and a close but apparently detached low mass companion are known. Initially, these systems were thought to be low accretion rate polars (LARPs; Reimers \& Hagen 2000), until Tout et al. (2008) suggested that, hidden among LARPs, there could be systems known as the prepolars or PREPs (Schwope et al. 2009), that emerged from common envelope evolution as close pairs but still await for gravitational radiation to bring them close enough for mass transfer to take place.

In this paper we introduce SMSS J160639.78-100010.7 (hereafter SMSS J1606-1000) which is a new highly magnetic white dwarf with a brown dwarf companion in a close orbit. This system is part of a selection of blue objects extracted from the SkyMapper survey (Onken et al. 2019). New optical spectroscopic and photometric data together with archival infrared and ultraviolet data are presented in Section 2 followed by our analysis of the binary parameters in Section 3.1, the photometric variations in Section 3.2, the character of the

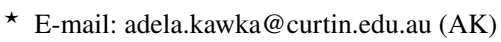

individual components of the system in Section 3.3, and of the system kinematics in Section 3.4. A discussion and our conclusions follow in Section 4.

\section{OBSERVATIONS}

We first obtained a spectrum of SMSS J1606-1000 with the FORS2 spectrograph attached to the Very Large Telescope at the European Southern Observatory on UT 2016 March 11. We used the 600B grism and set the slit width to 1 arcsec to provide a spectral coverage from 3500 to $6000 \AA$ at a resolution of $\approx 6 \AA$. The spectrum revealed a Zeeman-split hydrogen line spectrum in a strongly magnetic white dwarf with $\mathrm{H} \beta$ and weaker $\mathrm{H} \gamma$ emission lines. The emission lines do not show the effect of a magnetic field.

We followed-up with a series of spectra obtained with the WideField Spectrograph (WiFeS: Dopita et al. 2010) attached to the $2.3 \mathrm{~m}$ telescope at Siding Spring Observatory (SSO) on UT 2016 April 8, 2020 May 27 to 29, 2021 February 20, April 5, May 3, and June 19 . We used the B 3000 and R3000 gratings which cover a combined spectral range from 3500 to $9000 \AA$ during the 2016 April and 2020 May runs, and the $\mathrm{B} 3000$ and R7000 gratings with a coverage from 3500 to $7000 \AA ̊$ during all subsequent runs. We set the exposure times to $1800 \mathrm{~s}$ in the 12 exposures obtained on 2016 April 8 and 2020 May 27 and reduced the exposure times to $1200 \mathrm{~s}$ in all subsequent 48 exposures. Frequent NeAr arc spectra were obtained each night. The data were reduced using the PyWiFeS reduction pipeline (Childress et al. 2014). The pipeline generates three-dimensional data sets consisting of spatially resolved wavelength and flux calibrated spectra for 
Table 1. Astrometry and photometry of SMSS J1606-1000.

\begin{tabular}{|c|c|c|c|c|c|}
\hline \multicolumn{2}{|c|}{ Parameter } & \multicolumn{3}{|c|}{ Measurement } & Ref. \\
\hline \multicolumn{2}{|c|}{ RA (J2000) } & \multicolumn{3}{|c|}{$16^{\mathrm{h}} 06^{\mathrm{m}} 39^{\mathrm{s}} .78$} & 1 \\
\hline \multicolumn{2}{|c|}{ Dec (J2000) } & \multicolumn{3}{|c|}{$-10^{\circ} 00^{\prime} 10^{\prime \prime} 7$} & 1 \\
\hline \multicolumn{2}{|c|}{$\mu_{\sigma} \cos \delta\left(" \mathrm{yr}^{-1}\right)$} & \multicolumn{3}{|c|}{$-0.1446 \pm 0.0002$} & 2 \\
\hline \multicolumn{2}{|c|}{$\mu_{\delta}\left(" \mathrm{yr}^{-1}\right)$} & \multicolumn{3}{|c|}{$-0.0796 \pm 0.0001$} & 2 \\
\hline$\pi$ (mas) & & \multicolumn{3}{|c|}{$9.27 \pm 0.13$} & 2 \\
\hline \multicolumn{6}{|c|}{ Photometry } \\
\hline Band & Measurement & Ref. & Band & Measurement & Ref. \\
\hline$G$ & $17.944 \pm 0.003$ & 2 & $g$ & $17.993 \pm 0.067$ & 1 \\
\hline$b_{p}$ & $17.969 \pm 0.010$ & 2 & $i$ & $18.088 \pm 0.010$ & 1 \\
\hline$r_{p}$ & $17.754 \pm 0.025$ & 2 & $z$ & $18.138 \pm 0.063$ & 1 \\
\hline$F U V$ & $20.260 \pm 0.210$ & 3 & Y & $17.895 \pm 0.034$ & 4 \\
\hline$N U V$ & $18.841 \pm 0.047$ & 3 & $J$ & $17.387 \pm 0.033$ & 4 \\
\hline$u$ & $18.259 \pm 0.025$ & 1 & $H$ & $15.874 \pm 0.018$ & 4 \\
\hline$v$ & $18.145 \pm 0.028$ & 1 & $K$ & $15.243 \pm 0.022$ & 4 \\
\hline
\end{tabular}

References: (1) Onken et al. (2019); (2) Gaia Collaboration et al. (2020); (3) Morrissey et al. (2007); (4) McMahon et al. (2013).

each slitlet. We then extracted background-subtracted spectra from the slitlets that provided significant flux from SMSS J1606-1000.

We obtained a photometric time series of SMSS J1606-1000 with the $1.54 \mathrm{~m}$ telescope at La Silla on UT 2018 April 6. We used the $R$ filter and the series lasted $\approx 4.8 \mathrm{hr}$ with 125 exposures of $120 \mathrm{~s}$ each.

We collected ultraviolet photometric measurements from the Galaxy Evolution Explorer (GALEX: Morrissey et al. 2007), optical photometric measurements from SkyMapper (Onken et al. 2019) and infrared photometric measurements from the VISTA Hemisphere Survey (VHS: McMahon et al. 2013). These are listed in Table 1 together with photometric and astrometric measurements obtained by Gaia (Gaia Collaboration et al. 2020).

SMSS J1606-1000 is not a known X-ray source.

\section{ANALYSIS}

Prompted by the presence of Balmer emission lines in the FORS2 spectrum presumably emerging from or near the upper atmosphere of a cool companion, we proceed with a period analysis of the radial velocity and photometric measurements. Next, we present an analysis of the spectroscopic and photometric measurements of the system revealing an infrared excess attributed in part to the cool companion.

\subsection{Binary properties}

We measured the radial velocities using the $\mathrm{H} \alpha$ emission lines. The velocities were corrected for the solar system's barycentre motion and we fitted these with a sinusoidal function

$v(t)=\gamma+K \sin \left[2 \pi\left(t-T_{0}\right) / P\right]$

where $P$ is the orbital period, $\gamma$ is the systemic velocity, $K$ is the velocity semi-amplitude, and $T_{0}$ is the initial epoch of inferior conjunction of the cool companion. Fig. 1 shows a period analysis of the radial velocities showing that the orbital period is $P=92.0374 \pm 0.0002 \mathrm{~min}$. The velocity semi-amplitude is $141.2 \pm 3.2 \mathrm{~km} \mathrm{~s}^{-1}$ and the systemic velocity $-38.4 \pm 2.2 \mathrm{~km} \mathrm{~s}^{-1}$. However, the velocity measurements suffer from orbital smearing because the exposure times correspond to approximately one quarter of the orbital period. We corrected for this
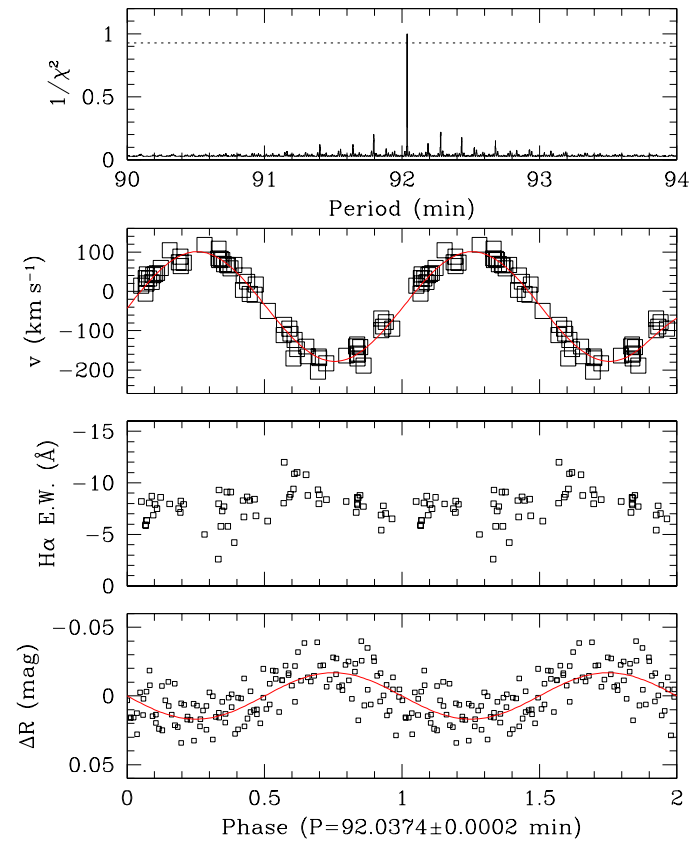

Figure 1. Top: Periodogram of the WiFeS $\mathrm{H} \alpha$ radial velocity measurements with the 90 percent confidence level shown with a dashed line. The phased radial velocity (second panel from top), $\mathrm{H} \alpha$ equivalent width (third panel), and R-band photometric measurements (bottom panel) are shown phased with the binary ephemeris.

effect by applying boxcar smoothing (full-width of 0.24 phase corresponding to the average exposure time divided by the orbital period) to the sinusoidal functions and by refitting the velocity measurements. We obtained a corrected velocity amplitude of $153.7 \pm 3.5 \mathrm{~km} \mathrm{~s}^{-1}$. A correction for surface variations of the Balmer emission was not included but it may amount to $\approx 8$ per cent if the Balmer emission is restricted to the hemisphere facing the white dwarf. Fig. 2 shows the WiFeS spectra averaged over four orbital phase bins. The $\mathrm{H} \beta$ emission traces the brown dwarf motion and absorption features trace the white dwarf motion. By cross-correlating the $\mathrm{H} \beta$ absorption features from 4700 to 4830 and from 4890 to $5100 \AA$ in the four phased spectra we find a white dwarf velocity semi-amplitude of $-38.9 \pm 27.5 \mathrm{~km} \mathrm{~s}^{-1}$ and an uncertain binary mass ratio $q=M_{\mathrm{BD}} / M_{\mathrm{WD}}=0.08-0.5$ which is only consistent with our spectroscopic analysis at the lower end, i.e., with a brown dwarf ( $q=0.08-0.10$, see section 3.3 ). The white dwarf orbit is poorly constrained by current data as the line profiles are possibly affected by surface field variations.

\subsection{Photometric variations}

The $R$ band photometric measurements show variations with a semiamplitude $\Delta m=0.017 \pm 0.002 \mathrm{mag}$ and a period of $93.3 \pm 5.9 \mathrm{~min}$ (Fig. 1, bottom panel). The photometric period is consistent with the orbital period. Maximum reprocessed light in a close pair involving a hot compact star with a cool tidally-locked companion should occur at the inferior conjunction of the hot primary $(\Phi=0.5)$. However, we measured maximum light at orbital quadrature $\Phi=0.75$ with a phase offset of only $-0.0007 \pm 0.0011$ implying that the photometric variations are probably due to a magnetic spot on the white dwarf surface 

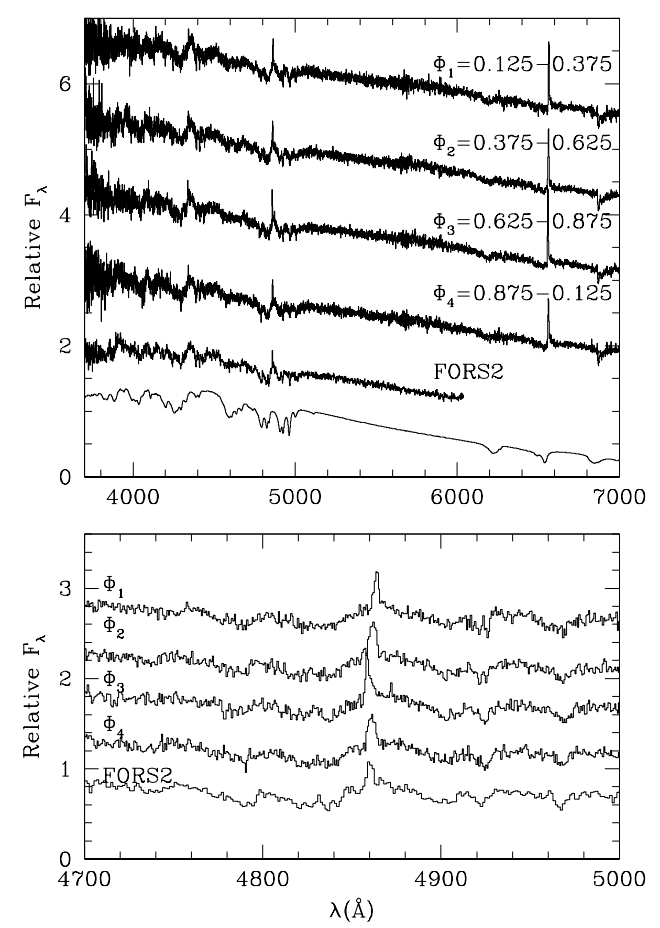

Figure 2. Top: WiFeS flux spectra binned at four consecutive orbital phases and FORS2 spectrum compared to a model spectrum (unlabeled) at $T_{\text {eff }}=$ $9600 \mathrm{~K}$ and $\log g=8.2$ with a dipolar field of $30 \mathrm{MG}$ (see Section 3.2). The Balmer emission spectrum at $\mathrm{H} \alpha, \mathrm{H} \beta$ and $\mathrm{H} \gamma$ is in evidence on top of the white dwarf absorption spectrum. Bottom: Same spectra but zoomed in around $\mathrm{H} \beta$ showing variability between the four phases.

assuming synchronous rotation, i.e., that the rotational period of the white dwarf is identical to the orbital period. Ellipsoidal variations could produce a peak at $\Phi=0.75$, but it would also produce a peak at $\Phi=0.25$ that is not observed. Photometric observations in multiple bands are needed to investigate further the source of the variations.

SMSS J1606-1000 was observed with GALEX over four separate visits in the $N U V$ and two visits in the $F U V$. We used GРнотоN (Million et al. 2016) to extract $N U V$ magnitudes from 4 separate observations to check for possible variability. The $N U V$ measurements show variability of up to 0.2 magnitudes but with only a 40 percent probability because of the large uncertainty in the individual measurements $(\sigma=0.11-0.13)$. The $F U V$ observations are very uncertain and do not provide any constraints on variability.

\subsection{Atmospheric and stellar parameters}

We have used the spectral energy distribution (SED) combined with the distance measurement from the Gaia parallax to constrain the white dwarf and companion properties, while accounting for possible cyclotron emission. We modeled the magnetic white dwarf using a prescription for the field distribution from Martin \& Wickramasinghe (1984) and Achilleos \& Wickramasinghe (1989). The visible surface was modeled using 450 elements co-added to provide a surfaceaveraged emergent spectrum. We utilized Stark broadened hydrogen line profiles with field-dependent line positions and strengths from Schimeczek \& Wunner (2014). For completeness, we added Zeemanshifted quasi-molecular Ly $\alpha$ opacities in the FUV spectral range with zero-field line opacities from Allard \& Koester (1992). The effective temperature and surface gravity of the white dwarf are $9600 \pm 300 \mathrm{~K}$ and $\log g=8.2 \pm 0.08$, respectively, corresponding to a mass of

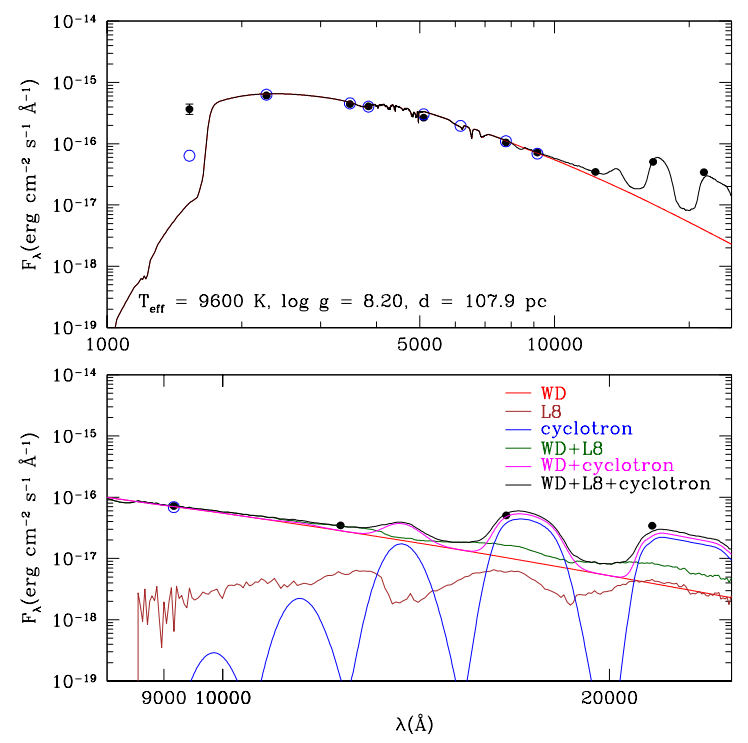

Figure 3. The spectral energy distribution of SMSS J1606-1000. The top panel shows photometric measurements compared to a white dwarf model spectrum at an effective temperature of $9600 \mathrm{~K}$ and surface gravity $\log g=8.2$ (in red) and the combined spectrum which includes contribution from the white dwarf, brown dwarf and cyclotron emission. The bottom panel shows the various flux contributions in the IR.

$0.72 \pm 0.05 M_{\odot}$ and, assuming no prior interactions, a cooling age of $9.7 \pm 0.6 \times 10^{8} \mathrm{yr}$ (Benvenuto \& Althaus 1999). The white dwarf progenitor mass is between $\approx 2.5 \mathrm{M}_{\odot}$ and $3.5 \mathrm{M}_{\odot}$ (Renedo et al. 2010) with a corresponding main-sequence lifetime ranging from $3 \times 10^{8}$ yr to $7 \times 10^{8}$ yr (Romero, Campos, \& Kepler 2015) for a total age of 1.3-1.6 Gyr which may fall short due to likely past interactions. We modeled the spectral Zeeman components and found that we could match the main features with a dipolar field of $30 \mathrm{MG}$ at an inclination of $65^{\circ}$ with an offset along the magnetic axis of +0.2 . Fig. 2 compares a model spectrum to four phase-binned WiFeS spectra obtained during the May 2020 run. The spectra show that the Zeeman-split Balmer lines vary as a function of the phase indicating that the magnetic field strength varies across the white dwarf surface.

Fig. 3 shows the SED of SMSS J1606-1000 comparing the flux contribution from the white dwarf, the companion and the cyclotron emission. We scaled the flux from the brown dwarf template on the absolute $H$ magnitude from Faherty et al. (2012) set at a distance of 107.9 pc. The SED near the VISTA- $J$ band constrains the companion to be a brown dwarf with a spectral type of about L8 (Testi et al. 2001) which corresponds to an effective temperature of $\sim 1500 \mathrm{~K}$ (Filippazzo et al. 2015). The GALEX/FUV flux is significantly higher than the predicted flux from the white dwarf models suggesting the effect of UV excess due to a hot spot on the white dwarf.

Fig. 4 shows available constraints on the secondary mass, radius and spectral type at an age of $1.5 \mathrm{Gyr}$ and up to $9 \mathrm{Gyr}$ (Baraffe et al. 2015). The Roche lobe radius was calculated following Eggleton (1983). We found that only a secondary star earlier than M5 would fill its Roche lobe. However, the SED analysis points toward an L8 brown dwarf secondary and no earlier than L2. Adopting an L8 type and accounting for a wide range of ages (1.5-9 Gyr), we estimate a secondary mass of $0.06-0.07 M_{\odot}$ or a mass ratio $q=0.08-0.1$, and a secondary radius $R_{2}=0.086-0.092 R_{\odot}$.

The brown dwarf accounts for some of the IR excess in the SED, and the remaining excess can only be explained by a hot spot with a cyclotron absorption spectrum. We have calculated the cyclotron 


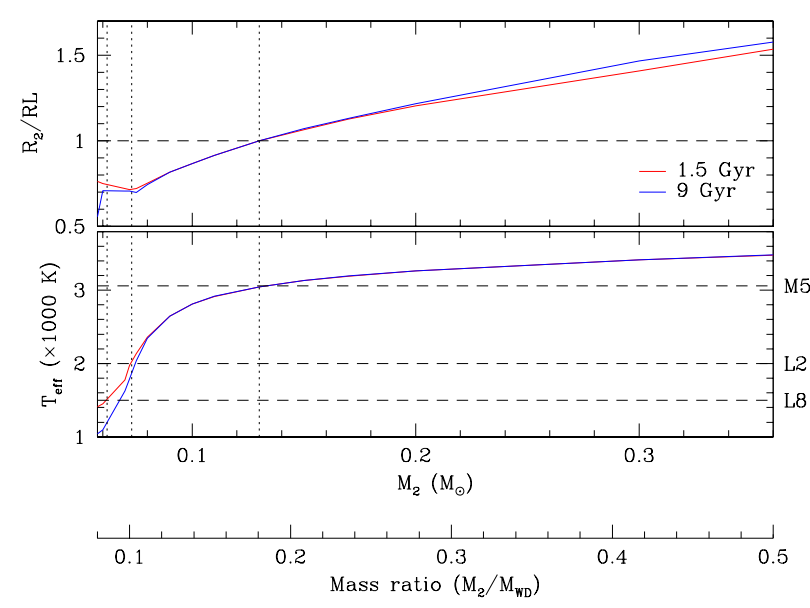

Figure 4. Top: Ratio of the secondary radius $\left(R_{2}\right)$ to the Roche lobe radius $(R L)$ at two different ages as a function of the secondary mass $\left(M_{2}\right)$ or, alternatively, the binary mass ratio assuming $M_{\mathrm{WD}}=0.72 M_{\odot}$. The rightmost vertical line marks a filled Roche lobe, and the middle and leftmost lines mark, respectively, the secondary mass upper limit and the preferred mass obtained from the SED analysis. Bottom: Corresponding effective temperatures $\left(T_{\text {eff }}\right)$ for the same relations depicted in the top panel showing the resulting constraints on the spectral type (horizontal lines).

spectrum following Chanmugam \& Dulk (1981) and Engelmann \& Curatolo (1973). To match the IR photometric measurements we used a thermal plasma of $k T=2.0 \mathrm{keV}$ with a thickness of $9 \times 10^{3} \mathrm{~cm}$, an electron density of $10^{16} \mathrm{~cm}^{-3}$, and a uniform magnetic field adjusted to $15.5 \mathrm{MG}$ at an inclination of $75^{\circ}$. The cyclotron emission region covers 0.04 per cent of the white dwarf's surface and is located near the weaker but more favourably inclined pole at $\approx 15 \mathrm{MG}$. Infrared spectroscopy is needed to disentangle the flux contribution from the brown dwarf and the cyclotron emission.

\subsection{Kinematics}

We calculated the Galactic velocity components relative to the local standard of rest using the distance, proper motion and systemic velocity following Johnson \& Soderblom (1987). The velocities of SMSS J1606-1000 were corrected for the Solar motion relative to the local standard of rest using $\left(U_{\odot}, V_{\odot}, W_{\odot}\right)=(11.10,12.24,7.25) \mathrm{km} \mathrm{s}^{-1}$ (Schönrich, Binney, \& Dehnen 2010). The Galactic velocity components of $(U, V, W)=(-32,-68,16) \mathrm{km} \mathrm{s}^{-1}$ place SMSS J1606-1000 in the thick disc (Soubiran, Bienaymé, \& Siebert 2003). Next, we calculated the eccentricity, $e=0.34$, and the $z$-component of the angular momentum, $L_{z}=1200 \mathrm{kpc} \mathrm{km} \mathrm{s}{ }^{-1}$, of the Galactic orbit using GALPY (Bovy 2015) where we used the MWPotential2014 Galactic potential. These properties place SMSS J1606-1000 in the thick disc following the classification of Pauli et al. (2006) and with an age of $\approx 9-10 \mathrm{Gyr}$ (Sharma et al. 2019) well in excess of the white dwarf age (1.3-1.6 $\left.\times 10^{9} \mathrm{yr}\right)$ derived assuming single star evolution. SMSS J1606-1000 must be an old system that experienced delays in the white dwarf cooling due to accretion.

\section{DISCUSSION AND CONCLUSIONS}

SMSS J1606-1000 belongs to a sub-class of close binaries consisting of a white dwarf paired with a very low mass companion. Such binaries exhibit a peak in the $\mathrm{M}$ dwarf companion distribution near spectral type M3.5 with a very steep decline at spectral types later
Table 2. Atmospheric and orbital parameters of SMSS J1606-1000.

\begin{tabular}{lc}
\hline Parameter & Measurement \\
\hline$T_{\text {eff,WD }}(\mathrm{K})$ & $9600 \pm 300$ \\
$\log g(\mathrm{cgs})$ & $8.2 \pm 0.08$ \\
Mass $\left(\mathrm{M}_{\odot}\right)$ & $0.72 \pm 0.05$ \\
$B_{\mathrm{WD}}(\mathrm{MG})$ & 30 \\
Period (d) & $0.06391487 \pm 0.00000014$ \\
$T_{0}(\mathrm{HJD})$ & $2458436.2144 \pm 0.0015$ \\
$K_{\mathrm{BD}}\left(\mathrm{km} \mathrm{s}^{-1}\right)$ & $141.2 \pm 3.2$ \\
$K_{\mathrm{BD}, \mathrm{corr}}\left(\mathrm{km} \mathrm{s}^{-1}\right)$ & $153.7 \pm 3.5$ \\
$\gamma\left(\mathrm{km} \mathrm{s}^{-1}\right)$ & $-38.4 \pm 2.2$ \\
\hline
\end{tabular}

than M5 (Ferrario 2012). The survey of Farihi, Becklin \& Zuckerman (2005) found, relative to this peak, $\approx 2-3$ times more $L$ dwarfs and $\approx$ 4-5 times more M6-M9 dwarfs in the field than among companions to white dwarfs. Despite more recent surveys with greater sensitivity to late type $\mathrm{M}$ dwarfs and brown dwarfs, very few of these systems have been detected (e.g Hogg et al. 2020). Furthermore, Carmichael et al. (2020) have reported that there are only 23 brown dwarfs transiting main-sequence stars with orbits within $10 \mathrm{AU}$ revealing that the 'brown dwarf desert' puzzle still persists. This phenomenon observed by Marcy \& Butler (2000) consists of a lack of brown dwarfs within $3 \mathrm{AU}$ of a main-sequence star. The descendants of these binaries, a white dwarf with a brown dwarf companion, are even more infrequent. If the white dwarf is magnetic, such a pairing becomes very rare. Only six magnetic systems with very late $\mathrm{M}$ type or brown dwarf companions are known (Table 3).

The component masses derived in the SED analysis of SMSS J1606-1000 resulted in a binary mass ratio $q=0.08-0.1$. We derived a Roche lobe radius $R_{L}=0.11-0.13 R_{\odot}$ for the brown dwarf. With a radius of only $0.086-0.092 R_{\odot}$ (Baraffe et al. 2015), the brown dwarf would fill at most 60 per cent of its Roche lobe. The system SMSS J1606-1000 appears detached.

In the case of SDSS J1212+0136, early studies suggested that the brown dwarf was under filling its Roche lobe and was more likely to be a PREP than a LARP (Schmidt et al. 2005; Farihi et al. 2008). However, the X-ray observations of Stelzer et al. (2017) strongly suggest that this system may be filling its Roche lobe and should be considered a LARP.

Other magnetic systems with a brown dwarf secondary are SDSS J1514+0744 and SDSS J1250+1549 (Breedt et al. 2012). The spectral type of the companion in IL Leo (=SDSS J1031+2028) (Schmidt et al. 2007; Parsons et al. 2021) has not been established yet. Finally, EF Eri was a persistent and bright X-ray source from its discovery in 1979 (Williams et al. 1979) until it entered a low accretion state in 1997 (Beuermann et al. 2000); its companion is probably an L or T dwarf (Beuermann et al. 2000; Howell et al. 2006).

Systems with very low mass companions could either be (i) polars in a prolonged low-state of accretion, like EF Eri, or (ii) detached binaries that will eventually undergo Roche lobe overflow, or (iii) old polars that have reached the orbital period minimum $P_{\text {orb }}$ for $\mathrm{CVs}$ and are evolving towards longer periods (period bouncers). Genuine PREPs, however, are expected to exhibit longer orbital periods (> 2.5 hrs, Ferrario, de Martino \& Gänsicke 2015) and this seems to be the case for most systems currently classified as PREPs, except for IL Leo (=SDSS J1031+2028). IL Leo has $P_{\text {orb }}=82 \mathrm{~min}$, a white dwarf with a field of $42 \mathrm{MG}$ and a companion of spectral type later than M6 and likely to under fill its Roche lobe (Schmidt et al. 2007).

If a binary is a PREP, the brown dwarf in the system should be in its original state. This hypothesis is supported by the observations 
Table 3. Magnetic systems with a late $M$ or brown dwarf secondary star

\begin{tabular}{lccrccc}
\hline Name & $\begin{array}{r}\mathrm{P}_{\text {orb }} \\
(\mathrm{min})\end{array}$ & $\begin{array}{c}\mathrm{M}_{\mathrm{WD}} \\
\left(\boldsymbol{M}_{\odot}\right)\end{array}$ & $\begin{array}{r}\mathrm{T}_{\text {eff }} \\
(\mathrm{K})\end{array}$ & $\begin{array}{c}B \\
(\mathrm{MG})\end{array}$ & type & Ref. \\
\hline $1606-1000$ & 92 & 0.72 & 9600 & 30 & $\mathrm{~L} 8$ & 1 \\
$1514+0744$ & 89 & $\ldots$ & 10000 & 36 & $\mathrm{~L} 3$ & 2 \\
$1212+0136$ & 88 & $>0.41$ & 9500 & $7-13$ & $\mathrm{~L} 5-8$ & $3,4,5$ \\
$1250+1549$ & 86 & $>0.42$ & 10000 & 20 & $\mathrm{M} 8$ & 2 \\
IL Leo & 82 & $>0.48$ & $<11000$ & 42 & $>\mathrm{M} 6$ & 6,7 \\
EF Eri & 81 & $>0.65$ & 9850 & $16-21$ & $>\mathrm{L} 4$ & $8,9,10$
\end{tabular}

References: (1) This work; (2) Breedt et al. (2012); (3) Schmidt et al. (2005); (4) Burleigh et al. (2006); (5) Farihi et al. (2008); (6) Parsons et al. (2021); (7) Schmidt et al. (2007); (8) Ferrario, Bailey, \& Wickramasinghe (1996); (9) Szkody et al. (2010); (10) Beuermann et al. (2000)

of Maxted et al. (2006) showing that the brown dwarf companion to the white dwarf WD 0137-349 was engulfed by the red giant but survived the experience unscathed.

However, if the brown dwarf belongs to a LARP or to a period bouncer, it was born as a hydrogen burning $\mathrm{M}$ dwarf that was peeled off by mass transfer leaving a degenerate sub-stellar object that can no longer burn fuel. Its structure and chemical composition (e.g., higher helium abundance and no lithium) would differ from those of a genuine brown dwarf.

The evolution of CVs is driven by angular momentum losses from the orbit. Because of mass transfer, both the mass of the secondary star and the orbital period decrease during evolution, but when the secondary's thermal time-scale and the mass transfer time-scale equal each other the period starts increasing. This period bounce occurs when $M_{2} \approx 0.07 \mathrm{M}_{\odot}$ (Paczynski 1981).

SMSS J1606-1000 is only the third known weakly- or noninteracting magnetic white dwarf plus brown dwarf binary after SDSS J1212+0136, SDSS J1514+0744. The properties of these systems are similar with orbital periods near 90 minutes and white dwarf effective temperatures near $10000 \mathrm{~K}$. SMSS J1606-1000 has a longest orbital period of the three. Based on the kinematics, we found that SMSS J1606-1000 is a very old system ( $>9$ Gyr) in the thick disc which excludes a PREP classification. The relatively long period and low mass ratio suggest that SMSS J1606-1000 is a period bouncer rather than a LARP.

A possible evolutionary scenario involves a wide main-sequence binary with a $2.5 M_{\odot}$ primary and $0.2 M_{\odot}$ secondary evolving through a CE phase after 0.8 Gyr. We adopted the low-metallicity $(Z=0.004)$ initial-to-final mass relations appropriate for the thick disc (Romero, Campos, \& Kepler 2015). The CE phase is followed by cycles of mass transfer and nova ejection of excess material from the binary which, finally, bounces off minimum period leaving it in its present state in $\approx 10 \mathrm{Gyr}: P_{\text {orb }}=0.064 \mathrm{~d}, M_{\mathrm{WD}}=0.72 M_{\odot}$, and $M_{\mathrm{BD}}=0.06 M_{\odot}$; The time spent in the $\mathrm{CV}$ phase, which maintains a steady white dwarf temperature $\left(\approx 10^{4} \mathrm{~K}\right)$ until it reaches minimum period, can be as long as $\approx 8$ Gyr (Belloni et al. 2018). Alternatively, a system with a lower mass primary $\left(1.5 M_{\odot}\right)$ that reaches the CE phase after 2 Gyr could achieve the same state if accreting white dwarfs are allowed to retain some of the transferred mass as proposed by Zorotovic, Schreiber, \& Gänsicke (2011).

\section{ACKNOWLEDGEMENTS}

This study is based on observations made with ESO telescopes at the La Silla Paranal Observatory under programme 097.D-0694. We thank Maruša Žerjal for her assistance with the WiFeS pipeline.

\section{DATA AVAILABILITY}

The FORS2 spectrum is publicly available at the ESO data archive (http://archive.eso.org/cms.html). The velocity measurements and the R-band photometric time series are available in electronic form.

\section{REFERENCES}

Achilleos N., Wickramasinghe D. T., 1989, ApJ, 346, 444

Allard N.F., Koester D., 1992, A\&A, 258, 464

Baraffe I., Homeier D., Allard F., Chabrier G., 2015, A\&A, 577, A42

Belloni D., Schreiber M. R., Zorotovic M., Iłkiewicz K., Hurley J. R., Giersz M., Lagos F., 2018, MNRAS, 478, 5626

Benvenuto O. G., Althaus L. G., 1999, MNRAS, 303, 30

Beuermann, K., Wheatley, P., Ramsay, G., Euchner, F., Gänsicke, B. T. 2000, A\&A, 354, L49

Bovy J., 2015, ApJS, 216, 29

Breedt E., Gänsicke B. T., Girven J., Drake A. J., Copperwheat C. M., Parsons S. G., Marsh T. R., 2012, MNRAS, 423, 1437

Burleigh M. R., et al., 2006, MNRAS, 373, 1416

Carmichael T. W., et al., 2020, AJ, 160, 53

Chanmugam G., Dulk G. A., 1981, ApJ, 244, 569

Childress M. J., Vogt F. P. A., Nielsen J., Sharp R. G., 2014, Ap\&SS, 349, 617

Dopita M., 2010, Ap\&SS, 327, 245

Eggleton P. P., 1983, ApJ, 268, 368

Engelmann F., Curatolo M., 1973, Nuclear Fusion, 13, 497

Faherty J. K., et al., 2012, ApJ, 752, 56

Farihi J., Becklin E. E., Zuckerman B., 2005, ApJ, 161, 394

Farihi, J., Burleigh M. R., Hoard D. W., 2008, ApJ, 674, 421

Ferrario L., Bailey J., Wickramasinghe D. T., 1996, MNRAS, 282, 218

Ferrario L., 2012, MNRAS, 426, 2500

Ferrario L., de Martino D., Gänsicke B. T., 2015, Space Sci. Rev., 191, 111

Filippazzo J. C., Rice E. L., Faherty J., Cruz K. L., Van Gordon M. M., Looper D. L., 2015, ApJ, 810, 158

Gaia Collaboration, Brown A. G. A., Vallenari A., Prusti T., de Bruijne J. H. J., Babusiaux C., Biermann M., 2020, A\&A, in press (arXiv:2012.0153)

Girven J., Gänsicke B. T., Steeghs D., Koester D., 2011, MNRAS, 417, 1210

Hogg M. A., et al., 2020, MNRAS, 498, 12

Howell, S. B. et al. 2006b, ApJ, 646, L65

Johnson D. R. H., Soderblom D. R., 1987, AJ, 93, 864

Martin B., Wickramasinghe D. T., 1984, MNRAS, 206, 407

Marcy G., Butler R., 2000, PASP, 112, 137

Maxted, P. F. L., Napiwotzki, R., Dobbie, P. D., Burleigh, M. R., 2006, Nature, 442,543

McMahon R. G., Banerji M., Gonzalez E., Koposov S. E., Bejar V. J., Lodieu N., Rebolo R., et al., 2013, Msngr, 154, 35

Million C., et al., 2016, ApJ, 833, 292

Morrissey P., et al., 2007, ApJS, 173, 682

Onken C. A., et al., 2019, PASA, 36, 33

Paczynski B., 1981, AcA, 31, 1

Pauli E.-M., Napiwotzki R., Heber U., Altmann M., Odenkirchen M., 2006, A\&A, 447, 173

Parsons, S. G., Gänsicke, B. T., Schreiber, M. R., Marsh, T. R., Ashley, R. P., Breedt, E., Littlefair, S. P., Meusinger, H., 2021, MNRAS, 502, 4305

Reimers D., Hagen H.-J., 2000, A\&A, 358, L45

Renedo I., Althaus L. G., Miller Bertolami M. M., Romero A. D., Córsico A. H., Rohrmann R. D., García-Berro E., 2010, ApJ, 717, 183

Romero A. D., Campos F., Kepler S. O., 2015, MNRAS, 450, 3708

Schimeczek C., Wunner, G., 2014, ApJS, 212, 26

Schmidt G. D., Szkody P., Silvestri N. M., Cushing M. C., Liebert J., Smith P. S., 2005, ApJL, 630, L173

Schmidt G. D., Szkody P., Henden A., Anderson S. F., Lamb D. Q., Margon B., Schneider D. P., 2007, ApJ, 654, 521

Schönrich R., Binney J., Dehnen W., 2010, MNRAS, 403, 1829

Schwope A. D., Nebot Gomez-Moran A., Schreiber M. R., Gänsicke B. T., 2009, A\&A, 500, 867 
Sharma S., et al., 2019, MNRAS, 490, 5335

Soubiran C., Bienaymé O., Siebert A., 2003, A\&A, 398, 141

Stelzer B., de Martino D., Casewell S. L., Wynn G. A., Roy M., 2017, A\&A, 598, L6

Steele P. R., Burleigh M. R., Dobbie P. D., Jameson R. F., Barstow M. A., Satterthwaite R. P., 2011, MNRAS, 416, 2768

Szkody, P., Mukadam, A., Gänsicke, B. T., et al. 2010, ApJ, 2010, 710, 64

Testi L., et al., 2001, ApJL, 552, L147

Tout C. A., Wickramasinghe D. T., Liebert J., Ferrario L., Pringle J., 2008, MNRAS, 387, 897

Williams G., Johns M., Price C., Hiltner A., Boley F., Maker S., Mook D. 1979, Nature, 81, 48

Zorotovic M., Schreiber M. R., Gänsicke B. T., 2011, A\&A, 536, A42

This paper has been typeset from a $\mathrm{T}_{\mathrm{E}} \mathrm{X} / \mathrm{L} \mathrm{AT} \mathrm{E}$ file prepared by the author. 